\title{
Epitaxially grown semiconducting hexagonal boron nitride as a deep ultraviolet photonic material
}

\author{
R. Dahal, J. Li, S. Majety, B. N. Pantha, X. K. Cao, J. Y. Lin, ${ }^{\text {a) }}$ and H. X. Jiang ${ }^{\text {b) }}$ \\ Department of Electrical and Computer Engineering, Texas Tech University, Lubbock, Texas 79409, USA
}

(Received 14 April 2011; accepted 4 May 2011; published online 24 May 2011)

\begin{abstract}
Hexagonal boron nitride (hBN) has emerged as an important material for various device applications and as a template for graphene electronics. Low-dimensional $\mathrm{hBN}$ is expected to possess rich physical properties, similar to graphene. The synthesis of wafer-scale semiconducting hBN epitaxial layers with high crystalline quality and electrical conductivity control has not been achieved but is highly desirable. Large area hBN epitaxial layers (up to 2 in. in diameter) were synthesized by metal organic chemical vapor deposition. P-type conductivity control was attained by in situ Mg doping. Compared to Mg-doped wurtzite AlN, which possesses a comparable energy band gap $(\sim 6 \mathrm{eV})$, dramatic reductions in $\mathrm{Mg}$ acceptor energy level and P-type resistivity (by about six to seven orders of magnitude) have been realized in hBN epilayers. The ability of conductivity control and wafer-scale production of $\mathrm{hBN}$ opens up tremendous opportunities for emerging applications, ranging from revolutionizing p-layer approach in III-nitride deep ultraviolet optoelectronics to graphene electronics. (C) 2011 American Institute of Physics.
\end{abstract}

[doi:10.1063/1.3593958]

Hexagonal boron nitride (hBN) possesses extraordinary physical properties such as ultrahigh chemical stability and band gap $\left(\mathrm{E}_{\mathrm{g}} \sim 6 \mathrm{eV}\right)$ (Ref. 1) and negative electron affinity. $^{2}$ Due to its unique layered structure and close inplane lattice match to graphene, low-dimensional hBN is expected to possess rich physical properties and could also be very useful as a template for graphene electronics. ${ }^{3,4}$ Due to its high band gap and in-plane thermal conductivity, $\mathrm{hBN}$ has been considered both as an excellent electrical insulator and thermal conductor. However, lasing action in deep ultraviolet (DUV) region $(\sim 225 \mathrm{~nm})$ by electron beam excitation was demonstrated in small hBN bulk crystals synthesized by a high pressure/temperature technique, ${ }^{5}$ raising its promise as a semiconducting material for realizing chip-scale DUV light sources/sensors. DUV $(\lambda<280 \mathrm{~nm})$ devices are highly useful in areas such as probing intrinsic fluorescence in a protein, equipment/personnel decontamination, and photocatalysis. Synthesizing wafer-scale semiconducting hBN epitaxial layers with high crystalline quality and electrical conductivity control has not been achieved but is highly desirable for the exploration of emerging applications. We report on the growth and basic properties of undoped and Mg-doped hBN epilayers grown on sapphire. Our results indicate that (a) hBN epitaxial layers exhibit outstanding semiconducting properties and (b) hBN is the material of choice for DUV optoelectronic devices.

Hexagonal BN epitaxial layers were synthesized by metal organic chemical vapor deposition using triethylboron source and ammonia $\left(\mathrm{NH}_{3}\right)$ as $\mathrm{B}$ and $\mathrm{N}$ precursors, respectively. Prior to epilayer growth, a $20 \mathrm{~nm} \mathrm{BN}$ or AlN buffer layer was first deposited on sapphire substrate at $800{ }^{\circ} \mathrm{C}$. The typical hBN epilayer growth temperature was about $1300{ }^{\circ} \mathrm{C}$. For the growth of $\mathrm{Mg}$-doped $\mathrm{hBN}$, biscyclopentadienyl-magnesium was transported into the re-

\footnotetext{
a) Electronic mail: jingyu.lin@ttu.edu.

${ }^{b)}$ Electronic mail: hx.jiang @ttu.edu.
}

actor during hBN epilayer growth. Mg-doping concentration in the epilayers used in this work was about $1 \times 10^{19} \mathrm{~cm}^{-3}$, as verified by secondary ion mass spectrometry (SIMS) measurement (performed by Charles and Evan). X-ray diffraction (XRD) was employed to determine the lattice constant and crystalline quality of the epilayers. Photoluminescence (PL) properties were measured by a DUV laser spectroscopy system. ${ }^{6}$ Hall-effect and standard Van der Pauw measurements were employed to measure the hole concentration and mobility and electrical conductivity. Seebeck effect (or hot probe) measurement was performed to further verify the conductivity type.

XRD $\theta-2 \theta$ scan shown in Fig. 1(a) revealed a $c$-lattice constant $\sim 6.67 \AA$, which closely matches to the bulk $c$-lattice constant of hBN $(c=6.66 \AA),{ }^{7-9}$ affirming that BN films are of single hexagonal phase. Figure 1(b) is the XRD rocking curve of the (002) reflection of a $1 \mu \mathrm{m}$ thick film. The observed linewidth is comparable to those of typical GaN epilayers grown on sapphire with a similar thickness. ${ }^{10}$ This signifies that these hBN epilayers are of high crystalline quality.

SIMS measurement results shown in Fig. 2(a) revealed that hBN epilayers have excellent stoichiometry. Figure 2(b)
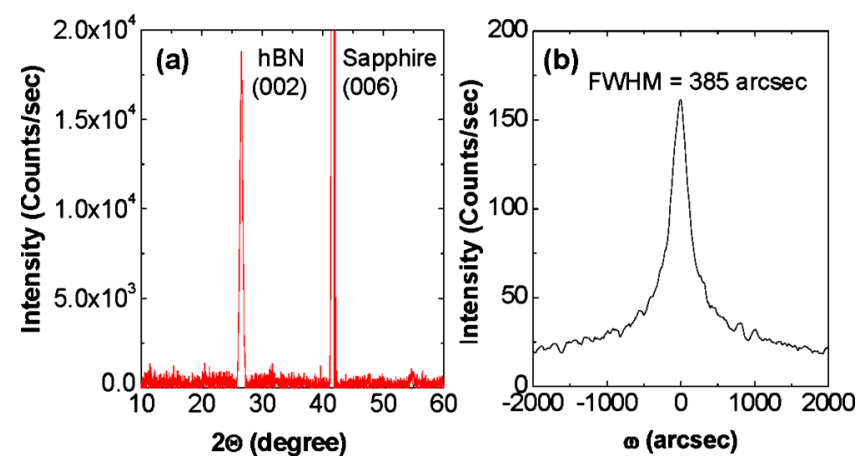

FIG. 1. (Color online) XRD measurement results of an hBN epilayer. (a) $\theta-2 \theta$ scan and (b) rocking curve of the (002) reflection. 

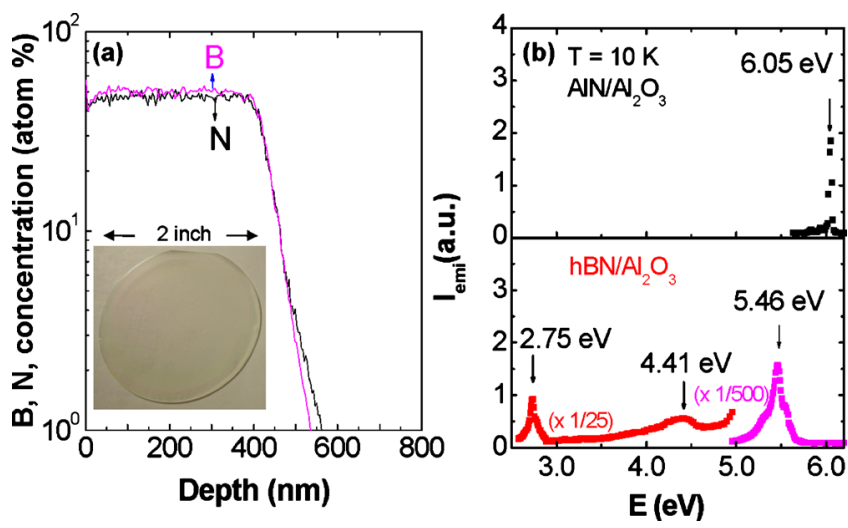

FIG. 2. (Color online) (a) SIMS measurement results of an hBN epilayer. The inset shows a micrograph of a 2 in. hBN epilayer wafer grown on sapphire substrate. (b) DUV PL emission spectrum of hBN and AlN epilayers measured at $10 \mathrm{~K}$. The PL emission of $\mathrm{hBN}$ and $\mathrm{AlN}$ was collected in the configuration of the electrical field of emission perpendicular $(\mathbf{E} \perp \mathbf{c})$ and parallel $(\mathbf{E} \| \mathbf{c})$ to the $c$-axis, respectively, controlled through the use of a polarizer in front of the monochromator.

is a low temperature PL spectrum, which exhibits a dominant emission line at $\sim 5.46 \mathrm{eV}$. Preliminary measurements on time-resolved PL seem to suggest that this emission line is most likely associated with a defect recombination. However, an interesting observation is that its emission intensity is about 500 times stronger than the dominant band-edge emission of a high quality AlN epilayer. ${ }^{6}$ This strong intensity may be related in part to the high band-edge optical absorption coefficient in $\mathrm{hBN}\left(>5 \times 10^{5} \mathrm{~cm}^{-1}\right){ }^{11}$

Today, AlGaN alloys have been the default choice for the development of DUV optoelectronic devices. Significant progress in nitride material and device technologies has been achieved. However, the most outstanding issue for realizing DUV light emitting diodes (LEDs) and laser diodes with high quantum efficiencies (QEs) is the low conductivity of p-type AlGaN. The resistivity of Mg-doped AlGaN increases with Al-content and becomes extremely high in Mg-doped AlN. As illustrated in Fig. 3(a), the $\mathrm{Mg}$ acceptor level $\left(\mathrm{E}_{\mathrm{A}}\right)$ in $\mathrm{Al}_{\mathrm{x}} \mathrm{Ga}_{1-\mathrm{x}} \mathrm{N}$ increases with $\mathrm{x}$, from about $170 \mathrm{meV}$ in $\mathrm{GaN}$ $\left(\mathrm{x}=0\right.$ with $\left.\mathrm{E}_{\mathrm{g}} \sim 3.4 \mathrm{eV}\right)$ to $510 \mathrm{meV}$ in $\mathrm{AlN}(\mathrm{x}=1$ with $\left.\mathrm{E}_{\mathrm{g}} \sim 6.1 \mathrm{eV}\right) .^{\mathrm{I}-15}$ Since the free hole concentration $(p)$ decreases exponentially with acceptor activation energy, $p \sim \exp \left(-\mathrm{E}_{\mathrm{A}} / \mathrm{kT}\right)$, an $\mathrm{E}_{\mathrm{A}}$ value around $500 \mathrm{meV}$ translates to only one free hole for roughly every $2 \times 10^{9}$ incorporated $\mathrm{Mg}$ impurities at room temperature. This leads to extremely
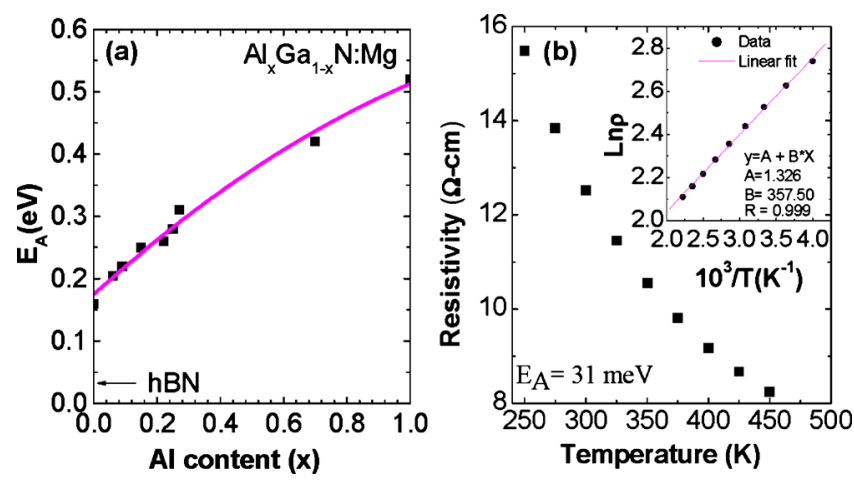

FIG. 3. (Color online) (a) $\mathrm{Mg}$ acceptor level $\left(\mathrm{E}_{\mathrm{A}}\right)$ in $\mathrm{AlGaN}$ and the arrow indicates $\mathrm{E}_{\mathrm{A}}$ in $\mathrm{hBN}: \mathrm{Mg}$. (b) p-type resistivity as a function of temperature of $\mathrm{hBN}: \mathrm{Mg}$.
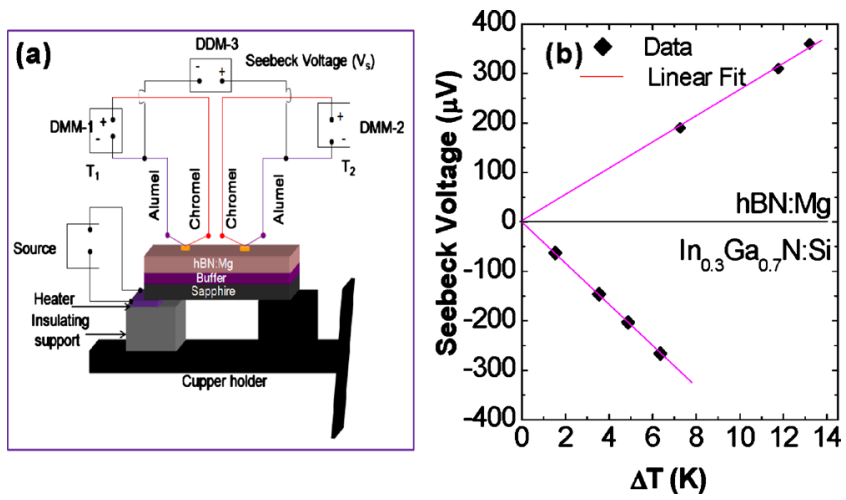

FIG. 4. (Color online) (a) Schematic of experimental setup of Seebeck effect measurement. (b) Seebeck coefficients of Mg-doped hBN (hBN:Mg) and n-type $\mathrm{In}_{0.3} \mathrm{Ga}_{0.7} \mathrm{~N}: \mathrm{Si}$ (with $\mathrm{n}=3 \times 10^{19} \mathrm{~cm}^{-3}$ and $\mu=90 \mathrm{~cm}^{2} / \mathrm{V} \mathrm{s}$ ).

resistive p-layers. For instance, an optimized $\mathrm{Mg}$-doped AlN epilayer has a typical "p-type resistivity" of $>10^{7} \Omega \mathrm{cm}$ at $300 \mathrm{~K}^{14}$ This causes an extremely low free hole injection efficiency into the quantum well active region and is a major obstacle for the realization of AlGaN-based DUV light emitting devices with high QE. Currently, the highest QE of AlGaN-based DUV $(\lambda<280 \mathrm{~nm})$ LED is around $3 \% .{ }^{16} \mathrm{It}$ should be noted that the deepening of the $\mathrm{Mg}$ acceptor level in $\mathrm{Al}_{\mathrm{x}} \mathrm{Ga}_{1-\mathrm{x}} \mathrm{N}$ with increasing $\mathrm{x}$ is a fundamental physics problem.

In contrast, as shown in Fig. 3, Mg-doped hBN (hB$\mathrm{N}: \mathrm{Mg}$ ) exhibits a p-type resistivity around $12 \Omega \mathrm{cm}$ at 300 $\mathrm{K}$ and the estimated $\mathrm{E}_{\mathrm{A}}$ value in $\mathrm{hBN}: \mathrm{Mg}$ is around $31 \mathrm{meV}$ based on the temperature dependent resistivity measurement. This value of $\mathrm{E}_{\mathrm{A}}$ is lower than previously determined acceptor levels ranging from $150-300 \mathrm{meV}$ in $\mathrm{BN}$ films containing mixed $\mathrm{cBN} / \mathrm{hBN}$ phases grown by evaporation and sputtering techniques. ${ }^{17-19}$ Hall-effect measurements revealed a free hole concentration $p \sim 1.1 \times 10^{18} \mathrm{~cm}^{-3}$ and mobility $\mu$ $\sim 0.5 \mathrm{~cm}^{2} / \mathrm{V} \mathrm{s}$. Based on the measured $\mathrm{E}_{\mathrm{A}}$ value of $31 \mathrm{meV}$ and Mg-doping concentration of $1 \times 10^{19} \mathrm{~cm}^{-3}$, the expected fraction of acceptor activation and $p$ value at $300 \mathrm{~K}$ would be about $30 \%$ and $3 \times 10^{18} \mathrm{~cm}^{-3}$, respectively. Thus, the measured and expected $p$ values are in a reasonable agreement. We expect the measured $p$ to be lower than the value estimated from acceptor activation since our hBN:Mg epilayers still possess appreciable concentrations of defects (including free hole compensating centers), as indicated in PL spectrum in Fig. 2.

In order to further confirm the conductivity type, we performed Seebeck effect (or hot probe) measurement on $\mathrm{hBN}: \mathrm{Mg}$ epilayers. Seebeck effect measurement is a wellestablished technique to distinguish between n-type and p-type conductivity of a semiconductor. ${ }^{20}$ A schematic illustration of the experimental setup for the Seebeck effect measurement is shown in Fig. 4(a). The sample was cut into a rectangular shape $\left(\sim 5 \times 20 \mathrm{~mm}^{2}\right)$. One end of the sample was placed on the sink while a heater was attached on the other end. On the surface of the sample, two thermocouples separated by $\sim 8 \mathrm{~mm}$ were attached. In-plane temperature gradient was created along the sample by the heater. The temperature gradient creates a voltage between the cold and hot ends due to the diffusion of thermally excited charged carriers. The direction of this induced potential gradient relative to the direction of the temperature gradient can be utilized to determine if the material is p- or n-type. The Seebeck 
voltage and temperature gradients were measured for $\mathrm{hB}$ $\mathrm{N}: \mathrm{Mg}$ against a standard n-type $\mathrm{In}_{0.3} \mathrm{Ga}_{0.7} \mathrm{~N}: \mathrm{Si}$ reference sample and the results are shown in Fig. 4(b). The Seebeck coefficient for $\mathrm{Si}$ doped $\operatorname{In}_{0.3} \mathrm{Ga}_{0.7} \mathrm{~N}$ was $S=\Delta \mathrm{V} / \Delta \mathrm{T}$ $+\mathrm{S}_{\text {Alumel }}=-42.2-18.5=-60.7 \mu \mathrm{V} / \mathrm{K}$ while for $\mathrm{Mg}$-doped $\mathrm{hBN}$ was $S=\Delta \mathrm{V} / \Delta \mathrm{T}+\mathrm{S}_{\text {Alumel }}=28.0-18.5=9.5 \mu \mathrm{V} / \mathrm{K}$. The sign reversal in $S$ over n-type $\mathrm{In}_{0.3} \mathrm{Ga}_{0.7} \mathrm{~N}$ : Si sample confirms unambiguously that hBN:Mg epilayers are p-type.

Further works are needed to further improve the overall material quality (and hence hole mobility) and understanding of the mechanisms for defect generation and elimination. Nevertheless, the dramatic reduction in $\mathrm{E}_{\mathrm{A}}$ and p-type resistivity (by about six to seven orders of magnitude) of $\mathrm{hBN}$ over AlN:Mg represents an exceptional opportunity to revolutionize p-layer approach and overcome the intrinsic problem of p-type doping in Al-rich AlGaN, thus potentially providing significant enhancement to the QE of DUV devices.

The work is support by DARPA-CMUVT grant \#FA2386-10-1-4165 (managed by Dr. John Albrecht). Jiang and Lin are grateful to the AT\&T Foundation for the support of Ed Whitacre and Linda Whitacre Endowed chairs.

${ }^{1}$ Y. Kubota, K. Watanabe, O. Tsuda, and T. Taniguchi, Science 317, 932 (2007).

${ }^{2}$ T. Sugino, C. Kimura, and T. Yamamoto, Appl. Phys. Lett. 80, 3602 (2002).

${ }^{3}$ D. Pacilé, J. C. Meyer, Ç. Ö. Girit, and A. Zettl, Appl. Phys. Lett. 92, 133107 (2008).

${ }^{4}$ L. Song, L. Ci, H. Lu, P. B. Sorokin, C. Jin, J. Ni, A. G. Kvashnin, D. G.
Kvashnin, J. Lou, B. I. Yakobson, and P. M. Ajayan, Nano Lett. 10, 3209 (2010).

${ }^{5}$ K. Watanabe, T. Taniguchi, and H. Kanda, Nat. Photonics 3, 591 (2009).

${ }^{6}$ K. B. Nam, J. Li, M. L. Nakarmi, J. Y. Lin, and H. X. Jiang, Appl. Phys. Lett. 84, 5264 (2004).

${ }^{7}$ V. Siklitsky, "boron nitride," http://www.ioffe.rssi.ru/SVA/NSM/ Semicond/BN/index.html.

${ }^{8}$ S. L. Rumyantsev, M. E. Levinshtein, A. D. Jackson, S. N. Mohammmad, G. L. Harris, M. G. Spencer, and M. S. Shur, in Properties of Advanced Semiconductor Materials GaN, AlN, InN, BN, SiC, SiGe, edited by M. E. Levinshtein, S. L. Rumyantsev, and M. S. Shur (Wiley, New York, 2001) pp. 67-92.

${ }^{9}$ R. W. Lynch and H. G. J. Drickamer, J. Chem. Phys. 44, 181 (1966).

${ }^{10} \mathrm{~S}$. Nakamura, G. Fasol, and S. J. Pearton, The Blue Laser Diode: The Complete Story (Springer, New York, 2000).

${ }^{11}$ A. Zunger, A. Katzir, and A. Halperin, Phys. Rev. B 13, 5560 (1976).

${ }^{12}$ J. Li, T. N. Oder, M. L. Nakarmi, J. Y. Lin, and H. X. Jiang, Appl. Phys. Lett. 80, 1210 (2002).

${ }^{13}$ M. L. Nakarmi, K. H. Kim, M. Khizar, Z. Y. Fan, J. Y. Lin, and H. X. Jiang, Appl. Phys. Lett. 86, 092108 (2005).

${ }^{14}$ M. L. Nakarmi, N. Nepal, C. Ugolini, T. M. Al Tahtamouni, J. Y. Lin, and H. X. Jiang, Appl. Phys. Lett. 89, 152120 (2006).

${ }^{15}$ Y. Taniyasu, M. Kasu, and T. Makimoto, Nature (London) 441, 325 (2006)

${ }^{16}$ C. Pernot, M. Kim, S. Fukahori, T. Inazu, T. Fujita, Y. Nagasawa, A. Hirano, M. Ippommatsu, M. Iwaya, S. Kamiyama, I. Akasaki, and H. Amano, Appl. Phys. Express 3, 061004 (2010).

${ }^{17}$ M. Lu, A. Bousetta, A. Bensaoula, K. Waters, and J. A. Schultz, Appl. Phys. Lett. 68, 622 (1996).

${ }^{18}$ K. Nose, H. Oba, and T. Yoshida, Appl. Phys. Lett. 89, 112124 (2006).

${ }^{19}$ B. He, W. J. Zhang, Z. Q. Yao, Y. M. Chong, Y. Yang, Q. Ye, X. J. Pan, J. A. Zapien, I. Bello, S. T. Lee, I. Gerhards, H. Zutz, and H. Hofsass, Appl. Phys. Lett. 95, 252106 (2009).

${ }^{20}$ B. Van Zeghbroeck, Principles of Semiconductor Devices (2007), Chap. 2. 